\title{
THE COMPARISON OF HEMODYNAMICS VARIABLES USING FLOTRAC (SENSOR) IN THE ENDOVASCULAR REPAIR OF ABDOMINAL (EVAR) AND THRACIC AORTIC ANEURYSM (TEVAR) USING DIFFERENT ANESTHETIC TECHNIQUES
}

\author{
S. Tsakiliotis ${ }^{1}$ \\ ${ }^{1}$ General Hospital Papageorgiou, Anaesthesiology, Thessaloniki, Greece \\ C. Pourzitaki ${ }^{2}$
}

${ }^{2}$ School of Health Sciences Aristotle University of Thessaloniki, ICU AHEPA University Hospital Faculty of Medicine-, Thessaloniki, Greece

V. Chasapidis ${ }^{3}$

${ }^{3}$ General Hospital Papageorgiou, Anaesthesiology, Thessaloniki, Greece

P. Douma ${ }^{4}$

${ }^{4}$ General Hospital Papageorgiou, Anaesthesiology, Thessaloniki, Greece

M. Doumparatzi ${ }^{5}$

${ }^{5}$ General Hospital Papageorgiou, Anaesthesiology, Thessaloniki, Greece

G. Tsaousi ${ }^{6}$

6-School of Health Sciences Aristotle University of Thessaloniki, ICU AHEPA University Hospital Faculty of Medicine, Thessaloniki, Greece

\section{Background and Aims:}

EVAR and TEVAR is being evaluated as an alternative therapeutic access to open aneurysm repair.

In this study we estimated hemodynamic parameters, like as cardiac output (CO), stroke volume (SV), and stroke volume variation (SVV) using FloTrac and compare these variables between spinal anaesthesia (EVAR) and general anaesthesia (TEVAR).

Methods:Thirty eight (EVAR) and thirty five patients (TEVAR) were enrolled. Intraoperative monitoring included pulse oxymetry, electrocardiogram with STsegment analysis and FloTrac (sensor) using Vigileo monitor (by Edwards Lifesiensces) in compination with invasive radial arterial pressure.

We recorded values of CO, SV, SVV, through the Vigileo device, at the following times:

(i) $5 \mathrm{~min}$ after application of spinal anesthesia or induction in general anaesthesia,

(ii) 3 min before the expansion of endograft,

(iii) 1 min after the deployment of central graft and

(iv) $1 \mathrm{~min}$ after the deployment of peripheral graft limb.

Differences between these specific times were evaluated by one sample t-test, Kolmogorov smirnov test, friedman test and pearson correlation $(p<0,05)$.

ResultsThere was significance difference in $\mathrm{CO}$ in comparison two anesthetic technique in times (i), (ii) and (iii).

Also there was significant difference in SV between two qroups in (i) and (ii) time period.

The same findings we had in SVV in (iii) and (iv) times.

Conclusions: The spinal anesthesia (EVAR) provides better hemodynamic stability in comparison withthe general anaesthesia (TEVAR).

Also there were differences between two groups in vascular tone(SVV), in (iii) and (iv) times, considering the need for low blood pressure in TEVAR technique. 